\title{
Assessment and management of venous thromboembolism risk during pregnancy and the puerperium (SAVE): The South African cohort
}

\author{
P Naidoo, ${ }^{1}$ BPharm, MB BCh, MMedSc (Pharmacology); R Mothilal, ${ }^{1}$ BPharm, MB BCh, MBA, DA (SA); \\ L C Snyman, ${ }^{2}$ BMedSci, MB ChB, MPraxMed, MMed (O\&G), FCOG (SA); on behalf of the SAVE South African participating doctors* \\ ${ }^{1}$ Sanofi South Africa \\ ${ }^{2}$ Department of Obstetrics and Gynaecology, School of Medicine, Faculty of Health Sciences, University of Pretoria and Kalafong Provincial \\ Hospital, Pretoria, South Africa \\ *SAVE participating doctors: Dr Sagie (Sagaren) Naidu, Dr Etienne Wilhelm Henn, Dr Shayhana Ganesh, Dr Sophie Mathijs, Dr Priya Soma-Pillay, \\ Prof. Leon Cornelius Snyman
}

Corresponding author: P Naidoo (poobalan.naidoo@sanofi.com)

\begin{abstract}
Background. Venous thromboembolism (VTE) is associated with significant morbidity and mortality. Pregnancy and the puerperium are hypercoagulable states and increase the risk of VTE. There is a paucity of South African (SA) data related to use of thromboprophylaxis during pregnancy and the puerperium.

Objectives. To evaluate local practice of VTE risk stratification among SA pregnant women and senior doctors' attitudes to VTE prophylaxis. Methods. This was a cross-sectional descriptive study of conveniently sampled sites in the private and public health sectors. Patients with confirmed pregnancy and an underlying medical condition were enrolled after giving informed consent. Assessments were made based on the participating doctors' questionnaires and case report forms. In essence, this was a local evaluation of a specific group of patients by a specific group of doctors.

Results. Two hundred and twenty patients were enrolled at six sites. In the participating doctors' opinion, 126/220 women assessed (57.2\%) were at risk of VTE during pregnancy and the postpartum period (information was missing for 1 woman during the postpartum period). Of the women at risk of VTE, 23/126 (18.3\%) were at high risk, 59/126 (46.8\%) at moderate risk and 44/126 (34.9\%) at low risk. Of the women identified as at risk of VTE, 104/127 (81.9\%) received some form of VTE prophylaxis; 94/127 (74.0\%) were at risk during pregnancy and 32/126 (25.4\%) during the postpartum period. Of those who received pharmacological treatment, 15/15 received low-molecular-weight heparin during pregnancy and before delivery and $87 / 100$ during the puerperium. Thirty-four patients received thromboprophylaxis for only 5 - 10 days after caesarean delivery, and 2 received mechanical thromboprophylaxis during pregnancy.

Conclusions. Doctors participating in the study were generally aware of VTE risk during pregnancy and the puerperium. Pharmacological thromboprophylaxis was the most commonly used intervention to reduce VTE risk. Mechanical thromboprophylaxis was underutilised. Adherence to VTE guidelines, specifically in terms of duration of thromboprophylaxis and its utilisation during pregnancy, was suboptimal.

S Afr Med J 2019;109(3):186-192. DOI:10.7196/SAMJ.2019.v109i3.13487
\end{abstract}

Venous thromboembolism (VTE) is a potentially life-threatening medical condition. Pregnancy induces a hypercoagulable state with an increase in coagulation factors and a decrease in natural anticoagulants, which increases the risk of deep-vein thrombosis (DVT) and pulmonary embolism (PE) in 0.5 - 2.2 per 1000 pregnancies. ${ }^{[1]}$ Furthermore, the puerperium is the time of maximal risk of pregnancy-associated VTE.

The use of thromboprophylaxis in patients at risk of VTE is of proven benefit, with low-molecular-weight heparin (LMWH) reducing VTE risk in medical and surgical patients by $60 \%$ and $70 \%$, respectively. ${ }^{[2]}$ No adequately powered randomised controlled trials have investigated the efficacy and safety of pharmacological prophylaxis in pregnancy and the puerperium. However, VTE pharmacological prophylaxis is advocated by both international ${ }^{[3]}$ and local guidelines, ${ }^{[4]}$ and is regarded as the standard of care in the appropriate patient.

The absolute risk for VTE in pregnancy is low, and risk stratification is required to determine which patients would benefit from thromboprophylaxis. ${ }^{[3]}$ There is a paucity of data on VTE risk stratification and thromboembolism prophylaxis in South African (SA) patients in general, ${ }^{[5]}$ and specifically during pregnancy and the puerperium.

\section{Objectives}

To evaluate local practices with regard to VTE risk stratification and thromboembolism prophylaxis during pregnancy and the puerperium.

\section{Methods}

This study was a sub-analysis of patients who participated in the SAVE global study, the primary objective of which was to estimate the proportion of women at risk of VTE during pregnancy and the postpartum period among all women attending prenatal and/ or obstetric centres. Secondary objectives included determining the proportion of women at risk receiving an effective type of VTE prophylaxis according to the American College of Chest Physicians ${ }^{[6]}$ and/or Royal College of Obstetricians and Gynaecologists (RCOG) $)^{[3]}$ guidelines, analysing factors that drive the decision to provide 
prophylaxis, and determining the overall proportion of patients receiving mechanical v. pharmacological prophylaxis. The criteria for VTE risk assessment (high risk, intermediate/moderate and lower risk) are set out in Table $1 .{ }^{[3]}$

\section{Study design and setting}

The SAVE study was a non-interventional, cross-sectional descriptive study conducted in 18 countries from December 2014 to October 2015. The primary study ${ }^{[7]}$ has been reported, and this article describes the SA cohort.

\section{Criteria for selection of doctors and patients}

A master list of doctors from SA was generated by the Sanofi clinical trials department. The list was based on the potential of the participating doctors to recruit the appropriate patients within the study timelines, and we attempted to obtain representation from both the SA public and private healthcare sectors. We then contacted each doctor on the master list and asked them to complete a site feasibility questionnaire to indicate their interest in the study and the number of patients they could potentially contribute to it.

Patients selected ${ }^{[7]}$ were women aged $>18$ years with objectively confirmed pregnancy, visiting for the first prenatal consultation or any other consultation during pregnancy, with an underlying medical condition, with/without the need for pre-partum hospitalisation for medical reasons, and who were willing to participate. Patients excluded were women with a VTE event in the preceding 4 months or those using concurrent antithrombotic therapy for other medical reasons.

\section{Information collected $^{[7]}$}

Information was collected via participating doctors' questionnaires and case report forms and included patient demographics, history, reason/s for consultation and VTE risk stratification. Information on the doctors included demographics, specialty, and their evaluation of VTE risk stratification and management.

\section{Sample size $\mathrm{e}^{[7]}$}

A detailed description of the statistical analysis can be found in the primary paper. ${ }^{[7]}$ The local sample size was selected based on numbers needed in the original SAVE study.

\section{Ethical considerations}

The study received approval from the local ethics committees (Pharma-Ethics and the University of Pretoria ethics committee), and the study participants gave signed informed consent.

\section{Results \\ Physician characteristics}

There were six study sites staffed with 6 medical doctors participating in the study. The majority of the doctors were aware of the existence of guidelines for VTE $(83.3,5 / 6)$ with 3 following the guidelines. Reasons for not following the guidelines included lack of time and 'other'. The questionnaire did not allow for description of the local guideline, which may have been interpreted as a local institutionally based guideline or national guideline.

\section{Patient disposition and characteristics}

Of a total of 224 patients screened, 220 were considered eligible to participate in the study. The 4 screen failures were based on the participating doctor's decision. Of the six study sites, 50\% were public sector sites and $50 \%$ private sector sites. A total of 90 and 130 patients were recruited from the private and public sector sites, respectively.

\begin{tabular}{|c|c|}
\hline Risk category & Risk factors \\
\hline \multicolumn{2}{|l|}{ Antenatal assessment } \\
\hline High risk & Any previous VTE other than a single event related to major surgery \\
\hline Intermediate/moderate risk & $\begin{array}{l}\text { Hospital admission; single previous VTE related to major surgery; high-risk thrombophilia and } \\
\text { no VTE; medical comorbidities, e.g. cancer, heart failure, active systemic lupus erythematosus, } \\
\text { inflammatory bowel disease or inflammatory polyarthropathy, nephrotic syndrome, type } 1 \text { diabetes } \\
\text { mellitus with nephropathy, sickle cell disease; current intravenous drug user; any surgical procedure, } \\
\text { e.g. appendicectomy; ovarian hyperstimulation syndrome (first trimester only) }\end{array}$ \\
\hline Lower risk ( $<3$ risk factors) & $\begin{array}{l}\text { Obesity (body mass index }>30 \mathrm{~kg} / \mathrm{m}^{2} \text { ); age }>35 \text { years; parity } \geq 3 \text {; smoker; gross varicose veins; current } \\
\text { pre-eclampsia; immobility, e.g. paraplegia, pelvic girdle pain with reduced mobility; family history } \\
\text { of unprovoked or oestrogen-provoked VTE in first-degree relative; low-risk thrombophilia; multiple } \\
\text { pregnancy; in vitro fertilisation/assisted reproductive technology; transient risk factors: dehydration/ } \\
\text { hyperemesis, current systemic infection, long-distance travel }\end{array}$ \\
\hline \multicolumn{2}{|l|}{ Postnatal assessment } \\
\hline High risk & $\begin{array}{l}\text { Any previous VTE; need for antenatal LMWH; high-risk thrombophilia; low-risk thrombophilia + } \\
\text { family history }\end{array}$ \\
\hline Intermediate/moderate risk & $\begin{array}{l}\text { Caesarean section in labour; body mass index } \geq 40 \mathrm{~kg} / \mathrm{m}^{2} \text {; readmission or prolonged admission } \\
\text { ( } \geq 3 \text { days) in the puerperium; any surgical procedure in the puerperium other than repair of } \\
\text { perineum; medical comorbidities, e.g. cancer, heart failure, active systemic lupus erythematosus, } \\
\text { inflammatory bowel disease or inflammatory polyarthropathy, nephrotic syndrome, type } 1 \text { diabetes } \\
\text { mellitus with nephropathy, sickle cell disease; current intravenous drug user }\end{array}$ \\
\hline $\begin{array}{l}\text { Intermediate/moderate risk } \\
\text { ( } \geq 2 \text { risk factors) and lower risk } \\
\text { ( }<2 \text { risk factors) }\end{array}$ & $\begin{array}{l}\text { Age }>35 \text { years; parity } \geq 3 \text {; smoker; elective caesarean section; family history of VTE; low-risk } \\
\text { thrombophilia; gross varicose veins; current systemic infection; immobility, e.g. paraplegia, pelvic } \\
\text { girdle pain with reduced mobility, long-distance travel; current pre-eclampsia; multiple pregnancy; } \\
\text { preterm delivery in this pregnancy ( }<37 \text { weeks); stillbirth in this pregnancy; mid-cavity rotational or } \\
\text { operative delivery; prolonged labour ( }>24 \text { hours); postpartum haemorrhage }\end{array}$ \\
\hline
\end{tabular}


Of the 220 patients entered into the study, 172 (78.2\%) were black. The mean (standard deviation (SD)) age of the cohort was 29.4 (5.9) years (Table 2). Thirty-two patients (14.5\%) were HIV-positive, and $98(44.5 \%)$ were obese (Table 3$)$. In the majority of cases $(n=169$, $76.8 \%$ ) the consultation was a routine visit (Table 4).

The majority of consultations were during pregnancy ( $n=183$, $83.2 \%)$, specifically the third trimester $(n=89,40.5 \%)$, with a mean (SD) gestation of 26.8 (9.1) weeks.

\section{Proportion and intensity of VTE risk}

According to the participating doctors, 126/220 patients (57.2\%) were at risk of VTE during pregnancy and postpartum (information was missing for 1 woman during the postpartum period); of the pregnant patients, $94 / 127(74.0 \%)$ were at risk, and of those in the in the puerperium, 32/126 (25.4\%) were at risk. When the risk was stratified by intensity, 9/32 postpartum patients (28.1\%) were at high risk. VTE risk stratification was missing for one patient. Table 5 sets out the risk stratification for women at risk of VTE during pregnancy and postpartum.

\section{VTE prophylaxis}

Of the 126 women at risk, $104(82.5 \%)$ (95\% confidence interval (CI) 74.08 - 88.16) were prescribed thromboprophylaxis, during pregnancy in $75 / 94$ cases $(79.8 \%)$ (95\% CI 70.25 - 87.37) and

\begin{tabular}{ll} 
Table 2. Patient demographics $(\mathbf{N = 2 2 0})$ & \\
\hline Age (years) & \\
Mean (SD) & $29.4(5.9)$ \\
Median & 29.0 \\
IQR & $25.0-33.0$ \\
Range & $19-44$ \\
Age in groups (years), $n(\%)$ & \\
$\leq 20$ & $12(5.5)$ \\
$20-30$ & $124(56.4)$ \\
$31-40$ & $76(34.5)$ \\
$41-50$ & $8(3.6)$ \\
$>50$ & 0 \\
$18-35$ & $179(81.4)$ \\
$>35$ & $41(18.6)$ \\
Ethnicity, $n(\%)$ & \\
White & $18(8.2)$ \\
Black & $172(78.2)$ \\
South Asian & $24(10.9)$ \\
Other & $6(2.7)$ \\
SD = standard deviation; IQR = interquartile range. &
\end{tabular}

\section{Table 3. Concomitant medical conditions $(N=220)$}

\begin{tabular}{ll}
\hline Medical condition & $\boldsymbol{n}(\%)$ \\
\hline Chronic lung disease & $5(2.3)$ \\
Chronic heart disease & $4(1.8)$ \\
Chronic neurological disease & $3(1.4)$ \\
Nephrotic syndrome (proteinuria $>3 \mathrm{~g} / 24 \mathrm{~h})$ & $6(2.7)$ \\
HIV infection & $32(14.5)$ \\
Active cancer & $1(0.5)$ \\
Short-term immobilisation $>3$ days & $16(7.3)$ \\
Reduced mobility $>10$ days & $7(3.2)$ \\
Obesity (body mass index $\left.>30 \mathrm{~kg} / \mathrm{m}^{2}\right)$ & $98(44.5)$
\end{tabular}

Table 4. Reasons for consultation/hospitalisation $(N=220)^{*}$

Table 4. Reasons

\begin{tabular}{ll} 
& $\boldsymbol{n}(\%)$ \\
\hline Routine visit & $169(76.8)$ \\
Intercurrent trauma & $1(0.5)$ \\
Intercurrent disease potentially necessitating & $18(8.2)$ \\
surgery/hospitalisation & \\
Intercurrent infection & $5(2.3)$ \\
Intercurrent inflammatory disease outbreak & $1(0.5)$ \\
Intercurrent jaundice/liver or biliary tract disease & $4(1.8)$ \\
Intercurrent renal function impairment & $7(3.2)$ \\
Gestational thrombocytopenia & $7(3.2)$ \\
Gestational anaemia & $13(5.9)$ \\
Maternal constitutional haemoglobin disease & $1(0.5)$ \\
Maternofetal alloimmunisation & $3(1.4)$ \\
Gestational diabetes mellitus & $28(12.7)$ \\
Pre-existing chronic hypertension, hypertension & $36(16.4)$ \\
in pregnancy & \\
Placenta-mediated pregnancy complication & $33(15.0)$ \\
Pre-eclampsia & $27(12.3)$ \\
Placental abruption & $6(2.7)$ \\
Intrauterine growth restriction & $11(5.0)$ \\
Premature rupture of the membranes & $2(0.9)$ \\
Fetal distress & $6(2.7)$ \\
Pregnancy loss & $8(3.6)$ \\
$\quad$ Gestational week of pregnancy loss occurrence & \\
$\quad$ Mean (SD) & $28.8(9.2)$ \\
$\quad$ Median & 28.5 \\
$\quad$ IQR & $27.0-36.0$ \\
$\quad$ Range & $9-38$ \\
Vaginal delivery & $10(4.5)$ \\
Caesarean delivery & $42(19.1)$ \\
Blood transfusion for postpartum haemorrhage & $11(5.0)$ \\
Postpartum wound infection & $3(1.4)$ \\
Caesarean infection & $3(1.4)$ \\
Surgical procedure (e.g. appendicectomy), pregnancy & $9(4.1)$ \\
or puerperium & \\
SD = standard deviation; IQR = interquartile range. \\
${ }^{*}$ atients could have more than one reason for consultation/hospitalisation. \\
& \\
& \\
&
\end{tabular}

\begin{tabular}{ll}
\multicolumn{2}{l}{ Table 5. Women at risk of VTE, stratified by risk intensity } \\
\hline Risk intensity & $\boldsymbol{n}(\%)$ \\
\hline All women $(N=126)$ & \\
$\quad$ Mild & $44(34.9)$ \\
Moderate & $59(46.8)$ \\
High & $23(18.3)$ \\
Missing & 1 \\
Women during pregnancy $(N=94)$ & \\
Mild & $40(42.6)$ \\
Moderate & $40(42.6)$ \\
High & $14(14.9)$ \\
Missing & 0 \\
Women during the postpartum period & \\
(N=32) & \\
Mild & $4(12.5)$ \\
Moderate & $19(59.4)$ \\
High & $9(28.1)$ \\
Missing & 1 \\
&
\end{tabular}


postpartum in an additional 29/33 (87.9\%) (95\% CI 71.80 - 96.60). Table 6 describes the patients receiving thromboprophylaxis during pregnancy and the puerperium.

During pregnancy and before delivery. Some patients had multiple reasons for non-prescription of thromboprophylaxis. The major reason for not prescribing thromboprophylaxis during pregnancy and before delivery was the fear of bleeding ( $n=24,40.7 \%)$, followed by a perception of increased risk associated with thromboprophylaxis (negative benefit/risk ratio) $(n=15,25.4 \%)$. Fourteen of 16 patients
(87.5\%) received pharmacological thromboprophylaxis, specifically LMWH, during pregnancy and before delivery. Two patients received only mechanical thromboprophylaxis during pregnancy and before delivery. Table 7 details VTE prophylaxis during pregnancy and delivery.

During the puerperium. One hundred and four patients (96.1\%) received prophylaxis during the puerperium (this figure includes those who had been at risk during pregnancy), with only $4(3.8 \%)$ not receiving therapy. The reasons for not receiving therapy were

\begin{tabular}{|c|c|}
\hline & $n(\%)\left(95 \% \mathrm{CI}^{*}\right)$ \\
\hline \multicolumn{2}{|c|}{ At-risk women receiving VTE prophylaxis $(N=127)$} \\
\hline No & $23(18.1)(11.84-25.92)$ \\
\hline Yes & $104(81.9)(74.08-88.16)$ \\
\hline \multicolumn{2}{|c|}{ At-risk women receiving VTE prophylaxis during pregnancy $(N=94)$} \\
\hline No & $19(20.2)(12.63-29.75)$ \\
\hline Yes & 75 (79.8) (70.25 - 87.37) \\
\hline \multicolumn{2}{|c|}{ At-risk women receiving VTE prophylaxis during the puerperium $(N=33)$} \\
\hline No & $4(12.1)(3.40-28.20)$ \\
\hline Yes & $29(87.9)(71.80-96.60)$ \\
\hline
\end{tabular}

\section{Table 7. Thromboprophylaxis during pregnancy and before delivery}

\begin{tabular}{|c|c|}
\hline & $n(\%)$ \\
\hline \multicolumn{2}{|l|}{ Reason for not prescribing thromboprophylaxis during pregnancy and before delivery ${ }^{\star}(N=59)$} \\
\hline No available proof & $14(23.7)$ \\
\hline Financial reason & $5(8.5)$ \\
\hline Fear of bleeding & $24(40.7)$ \\
\hline Negative benefit/risk ratio & $15(25.4)$ \\
\hline Other & $4(6.8)$ \\
\hline \multicolumn{2}{|l|}{ Thromboprophylaxis received during pregnancy and before delivery $(N=16)$} \\
\hline Pharmacological treatment & $14(87.5)$ \\
\hline Only mechanical treatment & $1(6.3)$ \\
\hline Both & $1(6.3)$ \\
\hline \multicolumn{2}{|l|}{ Type of pharmacological treatment during pregnancy and before delivery $(N=15)$} \\
\hline Unfractionated heparin & 0 \\
\hline LMWH & $15(100)$ \\
\hline NOACs & 0 \\
\hline Aspirin & $2(13.3)$ \\
\hline Other & 0 \\
\hline \multicolumn{2}{|l|}{ Duration of pharmacological treatment during pregnancy and before delivery $(N=15)$} \\
\hline Only third trimester & 0 \\
\hline Second and third trimester & 0 \\
\hline From now until delivery & $14(93.3)$ \\
\hline Only during the course of the intercurrent infection & 0 \\
\hline Only during the course of the inflammatory outbreak & 0 \\
\hline Only during the course of the intercurrent urgent surgery & 0 \\
\hline Only during the course of the intercurrent immobilisation & 0 \\
\hline Only during the course of the intercurrent hospitalisation & $1(6.7)$ \\
\hline Hospitalisation needed & 0 \\
\hline From now until delivery + hospitalisation needed & 0 \\
\hline Only during the course of the intercurrent urgent surgery + hospitalisation needed & 0 \\
\hline Only during the course of the intercurrent hospitalisation + hospitalisation needed & 0 \\
\hline If hospitalisation needed during pregnancy, thromboprophylaxis to be continued after hospital discharge $(N=0)$ & $\mathrm{n} / \mathrm{a}$ \\
\hline
\end{tabular}




\section{Table 8. Thromboprophylaxis during the puerperium}

Reason for not prescribing thromboprophylaxis during puerperium ${ }^{\star}(N=4), n(\%)$

No available proof

Financial reason

Fear of bleeding

$1(25.0)$

Negative benefit/risk ratio

$1(25.0)$

Other

0

Thromboprophylaxis received during puerperium $(N=100), n(\%)$

Pharmacological treatment

Only mechanical treatment

0

Both

Type of pharmacological treatment during puerperium ${ }^{\dagger}(N=100), n(\%)$

Unfractionated heparin

LMWH

NOACs

Aspirin

Thromboprophylaxis for 5 - 10 days post caesarean section

Type of unfractionated heparin during puerperium $(N=5), n(\%)$

Low dose

Intermediate dose

Weight-adjusted dose

Type of LMWH during puerperium ( $N=87), n(\%)$

Low dose

Intermediate dose

Weight-adjusted dose

Beginning of pharmacological treatment during puerperium (postpartum day) $(N=99)$

\section{Missing}

Mean (SD)

Median

1.0

IQR

Range

Duration of pharmacological treatment during puerperium $(N=100), n(\%)$

2 weeks post partum

4 weeks post partum

6 weeks post partum

12 weeks post partum

5 - 10 days post caesarean section

5 - 10 days post surgery

During the course of the intercurrent infection

During the course of the intercurrent immobilisation

Only during the course of the intercurrent hospitalisation

2 weeks post partum + hospitalisation needed

4 weeks post partum + hospitalisation needed

6 weeks post partum + hospitalisation needed

5 - 10 days post caesarean section + hospitalisation needed

Only during the course of the intercurrent hospitalisation + hospitalisation needed

If hospitalisation needed during puerperium, thromboprophylaxis to be continued after hospital discharge $(N=15), n(\%)$ 
'No available proof' $(n=2)$, i.e. the investigator does not consider thromboprophylaxis in pregnancy as 'valid' and 'recognised', fear of bleeding $(n=1)$, and perception of increased risk associated with thromboprophylaxis (negative benefit/risk ratio) $(n=1)$. Seventy patients (70.0\%) received pharmacological thromboprophylaxis and 30 (30.0\%) both pharmacological and mechanical prophylaxis in the puerperium. LMWH was the most commonly used pharmacological prophylaxis $(n=87,87 \%)$, with new oral anticoagulants (NOACs) (non-vitamin $\mathrm{K}$ antagonists) in 8 (8\%). Of note, 34 (34\%) received prophylaxis for only 5 - 10 days after caesarean section. Table 8 details VTE prophylaxis during the puerperium.

\section{Risk factors determining prophylaxis}

SA Asian women $(n=24)$ were more likely to be perceived to be at risk of developing VTE than white women (Wald $\chi^{2} p$-value 0.0316 ; odds ratio estimate 13.143; 95\% CI 1.255 - 137.667). Drivers for VTE thromboprophylaxis included pre-eclampsia, caesarean delivery and immobility (Table 9).

\section{Discussion}

Drivers for VTE thromboprophylaxis included pre-eclampsia, caesarean delivery and immobility. The relatively low use of VTE thromboprophylaxis during pregnancy is concerning. Clinicians cited fear of bleeding and perception of an increased risk associated with thromboprophylaxis (negative benefit/risk ratio) during pregnancy as the major deterrents to using pharmacological agents during pregnancy. However, mechanical thromboprophylaxis was underutilised, with only 2 patients receiving this during pregnancy. The RCOG Green-top guideline ${ }^{[3]}$ recommends the use of mechanical thromboprophylaxis for patients with a contraindication to LMWH. This is a grade $\mathrm{D}$ recommendation based on relatively less robust data. Mechanical thromboprophylaxis has no bleeding risk, and clinicians would therefore have been expected to use it in patients at high risk of bleeding diathesis. Non-availability of DVT stockings in the public health sector may have contributed to this finding.
Research is required to determine how clinicians stratify bleeding risk during pregnancy.

The majority of the patients received VTE prophylaxis for only 5 - 10 days after delivery, which is not aligned with current guidelines. According to the RCOG Green-top guideline, ${ }^{[3]}$ thromboprophylaxis should be continued for 6 weeks in high-risk women and for 10 days in those at intermediate risk. Short duration of thromboprophylaxis may result in a residual risk of VTE, with potential catastrophic consequences. Unfortunately the study did not elucidate the reasons for inadequate duration of thromboprophylaxis.

LMWH was the preferred pharmacological agent for VTE prophylaxis. Only $8 \%$ received a non-vitamin $\mathrm{K}$ oral anticoagulant in the postpartum period. LMWH is considered the standard of care locally and internationally. Non-vitamin $\mathrm{K}$ antagonists were used in the minority of patients, probably because the public sector sites did not have access to these drugs and they should not be used during pregnancy and lactation ${ }^{[3]}$ because their safety and efficacy during this period have not been established.

Snyman and Potgieter ${ }^{[8]}$ investigated the VTE risk profile and thromboprophylaxis in 109 patients undergoing gynaecological surgery in a single-centre academic unit. Only $5 \%$ of study participants received the correct VTE prophylaxis. Direct comparisons between their study ${ }^{[8]}$ and the present study cannot be made because they evaluated VTE in different groups of patients, i.e. gynaecological v. obstetric, and the doctors prescribing prophylaxis were unaware that they were being audited. Nevertheless, the present study had a higher proportion of patients receiving appropriate therapy. The clinicians who took part in the study are senior clinicians working in clinical trial sites and are therefore likely to be up to date with respect to guidelines. Furthermore, the study itself and the knowledge that they would be audited may have motivated them to better understand VTE risk stratification and management. These clinicians probably reflect the current best practices in SA.

HIV infection is increasingly being considered a prothrombotic state. ${ }^{[9]}$ The prevalence of HIV-positive patients in this study (14.5\%)

Table 9. Significant variables in univariate analysis

\begin{tabular}{|c|c|c|c|c|c|}
\hline \multirow[b]{2}{*}{ Variable } & \multirow[b]{2}{*}{ Modality } & \multirow[b]{2}{*}{ Reference } & \multirow[b]{2}{*}{ Wald $\chi^{2} p$-value } & \multicolumn{2}{|c|}{ OR } \\
\hline & & & & Estimate & $95 \% \mathrm{CI}$ \\
\hline Antithrombin deficiency & Unknown & No & 0.0002 & 0.056 & $0.012-0.251$ \\
\hline Caesarean delivery & Yes & No & 0.0142 & 6.562 & $1.460-29.505$ \\
\hline Emergency caesarean delivery & Yes & No & 0.0910 & 5.902 & $0.753-46.238$ \\
\hline \multirow[t]{3}{*}{ Ethnicity } & Black & Caucasian & 0.2404 & 2.222 & $0.586-8.429$ \\
\hline & Other* & Caucasian & * & * & \\
\hline & South Asian & Caucasian & 0.0316 & 13.143 & $1.255-137.667$ \\
\hline Factor V Leiden & Unknown & No & 0.0003 & 0.063 & $0.014-0.283$ \\
\hline Gross varicose veins & Yes & No & 0.0783 & 0.370 & $0.122-1.119$ \\
\hline Immobility & Yes $^{*}$ & No & * & * & \\
\hline Persistent beta-2-glycoprotein 1 antibodies & Unknown & No & 0.0003 & 0.064 & $0.014-0.288$ \\
\hline \multirow[t]{2}{*}{ Persistent lupus anticoagulant } & Unknown & No & 0.0002 & 0.059 & $0.013-0.267$ \\
\hline & Yes $^{*}$ & No & * & * & \\
\hline Persistent moderate/high-titre anticardiolipin & Unknown & No & 0.0003 & 0.061 & $0.014-0.274$ \\
\hline antibodies & Yes $^{*}$ & No & * & * & \\
\hline Pre-eclampsia & Yes & No & 0.0571 & 7.332 & $0.942-57.092$ \\
\hline Protein $\mathrm{C}$ deficiency & Unknown & No & 0.0002 & 0.056 & $0.012-0.251$ \\
\hline Protein S deficiency & Unknown & No & 0.0002 & 0.056 & $0.012-0.251$ \\
\hline Prothrombin gene $20210 \mathrm{~A}$ allele & Unknown & No & 0.0003 & 0.063 & $0.014-0.283$ \\
\hline
\end{tabular}


is lower than the prevalence of $\sim 29.7 \%$ in antenatal clinics in SA. ${ }^{[10]}$ These figures may differ because the antenatal clinic data only include public sector patients.

Our cohort had a high prevalence of obesity, with 98 women (44.5\%) having a body mass index $>30 \mathrm{~kg} / \mathrm{m}^{2}$. SA has the highest prevalence of obesity in sub-Saharan Africa, which may be attributed in part to rapid socioeconomic transition. ${ }^{[11]}$ Obesity complicates the use of pharmacological agents, with the possibility that altered pharmacokinetics may require dose adjustments. In addition, obesity itself is a risk factor for VTE.

\section{Study limitations}

The study was limited by the small sample of clinical trial sites (six sites) and the fact that the participating doctors were relative experts. Owing to the shortage of obstetricians and gynaecologists in SA, the majority of patients are treated by non-specialists whose adherence to guidelines may be less than ideal. The study may not reflect management of VTE risk during pregnancy and the puerperium in SA as a whole, which is dominated by a public sector characterised by skill shortages and high patient numbers. The study sites were all urban centres and therefore do not reflect rural practice. Despite its limitations, however, the study provides valuable insights into VTE risk stratification and thromboprophylaxis.

\section{Conclusions}

The doctors participating in the study were in general aware of VTE risk during pregnancy and the puerperium. However, VTE thromboprophylaxis during pregnancy could be improved. Clinicians cited fear of bleeding and a perception of increased risk associated with thromboprophylaxis (negative benefit/risk ratio) as reasons for not using pharmacological thromboprophylaxis during pregnancy, but still underused mechanical thromboprophylaxis. Patients in this study did not receive the recommended duration of thromboprophylaxis. Further research and novel methods are required to improve VTE prophylaxis during pregnancy and the puerperium.

We suggest the development of SA guidelines for the management of VTE risk during pregnancy or adoption of the current international guidelines (e.g. RCOG). Education of all relevant healthcare workers is needed regarding VTE risk stratification and management during pregnancy.

\section{Declaration. None.}

Acknowledgements. The authors thank the doctors and patients who participated in the study.

Author contributions. PN wrote the first draft, critically evaluated subsequent versions of the manuscript and granted permission to publish. PM critically evaluated all versions of the manuscript and granted permission to publish. LCS was the national study co-ordinator, critically evaluated all versions of the manuscript and granted permission to publish.

Funding. Sanofi funded all aspects of the study, including the article processing fee.

Conflicts of interest. PN and RM are Sanofi employees. Sanofi manufactures a low-molecular-weight heparin (enoxaparin). LCS has received speaking honoraria from Sanofi.

1. Villani M, Ageno W, Grandone E, Dentali F. The prevention and treatment of venous thromboembolism in pregnancy. Expert Rev Cardiovasc Ther 2017;15(5):397-402. https://doi.org/10.1080/14779072.201 7.1319279

2. National Institute of Health and Care Excellence. Venous thromboembolism: reducing the risk: Reducing the risk of venous thromboembolism (deep vein thrombosis and pulmonary embolism) in patients admitted to hospital. NICE clinical guideline 92, 2015. https://www.nice.org.uk/guidance/ cg92 (accessed 1 June 2017).

3. Royal College of Obstetricians and Gynaecologists. Reducing the risk of thrombosis and embolism during pregnancy and the puerperium. Green-top Guideline No. 37a, 2015. https://www.rcog.org.uk/ globalassets/documents/guidelines/gtg-37a.pdf (accessed 1 May 2017).

4. Jacobson BF, Louw S, Büller H, et al. Venous thromboembolism: Prophylactic and therapeutic practice guideline. S Afr Med J 2013;103(4 Pt 2):261-267. https://doi.org/10.7196/SAMJ.6706

5. Danwang C, Temgoua MN, Agbor VN, Tankeu AT, Noubiap JJ. Epidemiology of venous thromboembolism in Africa: A systematic review. J Thromb Haemost 2017;15(9):1770-1781. https:/ doi.org/10.1111/jth.13769

6. Bates SM, Greer IA, Middeldorp S, et al. VTE, thrombophilia, antithrombotic therapy, and pregnancy: Antithrombotic Therapy and Prevention of Thrombosis, 9th ed.: American College of Chest Physicians Evidence-Based Clinical Practice Guidelines. Chest 2012;141(2 Suppl):e691S-e736S. https://doi. org/10.1378/chest.11-2300

7. Gris J-C, Aoun J, Rzaguliyeva L, et al.; SAVE Study Group. Risk Assessment and Management of Venous Thromboembolism in Women during Pregnancy and Puerperium (SAVE): An international, cross-sectional study. TH Open 2018;02(02):e116-el30. https://doi.org/10.1055/s-0038-1635573

8. Snyman LC, Potgieter J. Venous thromboembolism: Risk profile and management of prophylaxis in gynaecological surgery patients. S Afr J Obstet Gynaecol 2014;20(3):76-79. http://www.sajog.org.za/ index.php/SAJOG/article/view/490/472 (accessed 1 May 2017).

9. Eyal A, Veller M. HIV and venous thrombotic events. S Afr J Surg 2009;47(2):54-56

10. National Department of Health, South Africa. The National Antenatal Sentinel HIV Prevalence Survey National Department of Health, South Africa. The National Antenatal Sentinel HIV Prevalence Survey,
South Africa, 2013. https://www.health-e.org.za/wp-content/uploads/2016/03/Dept-Health-HIVSouth Africa, 2013. https://www.health-e.o
High-Res-7102015.pdf (accessed 1 May 2017).

11. Sartorius B, Veerman LJ, Manyema M, Chola L, Hofman K. Determinants of obesity and associated population attributability, South Africa: Empirical evidence from a national panel survey, 2008 - 2012 PloS One 2015;10(6):e0130218. https://doi.org/10.1371/journal.pone.0130218

Accepted 21 August 2018 